\title{
To tell or not to tell? A systematic review of ethical reflections on incidental findings arising in genetics contexts
}

\author{
Gabrielle M Christenhusz $^{\star, 1}$, Koenraad Devriendt ${ }^{2}$ and Kris Dierickx ${ }^{1}$ \\ Any test that produces visual images or digital or genetic sequences will tend to produce incidental findings because more will \\ be visible than what was originally sought. We conducted a systematic review of the ethical reasons presented in the literature \\ for and against the disclosure of incidental findings arising in clinical and research genetics contexts. A search of electronic \\ databases resulted in 13 articles included for systematic review. Articles presented reasons for and against disclosure, and \\ reasons for proceeding with caution when making decisions about disclosure. One major recommendation of the reviewed \\ articles is in favor of qualified disclosure: incidental findings with confirmed clinical utility where there is the possibility of \\ treatment or prevention should be disclosed, with exceptions. A second type of recommendation is that disclosure should \\ proceed with caution, especially in the context of new genetic technologies and genetic testing involving minors. It is also \\ recommended that the number of possible incidental findings be limited even before genetic testing is carried out. Such a \\ policy, which we advocate, would show preference for non-disclosure.
}

European Journal of Human Genetics (2013) 21, 248-255; doi:10.1038/ejhg.2012.130; published online 27 June 2012

Keywords: incidental findings; ethics; research; clinical genetics; whole genome sequencing

\section{INTRODUCTION}

Incidental findings (IFs) can arise in all medical contexts, though they have been most frequently reported in neuroimaging, oncology and genetics settings. ${ }^{1}$ Examples include a brain aneurysm in a healthy control subject involved in neuroimaging research, ${ }^{1}$ a malignant skin tumor discovered during a woman's routine breast cancer screening, ${ }^{2}$ and learning that someone is of higher risk of Alzheimer's disease when they present at a genetics clinic wanting to know if there is a genetic cause for their cardiac condition. ${ }^{3}$ IFs have been defined as findings having potential health or reproductive importance for an individual, discovered in the course of conducting a particular study (in research, clinical care or screening) but beyond the aims of that study. ${ }^{1}$

In recent years, much has been published on IFs in general and IFs arising in imaging contexts. ${ }^{1,4-6}$ There is yet to be a systematic overview of IFs arising in genetics contexts. This gap in the research is not because IFs do not occur in genetics contexts. For example, a newborn screening program looking for infants with sickle cell disorder will also identify carriers of the disease, though this is not the purpose of the screening. ${ }^{7}$ Research identifying new disease genes in linkage studies that depend on accurately defining family pedigrees can uncover misattributed paternity, another example of an $\mathrm{IF}^{8}$ In clinical care, array-CGH conducted to identify the genetic cause for a case of congenital heart disease could reveal CNVs related to other disorders. IFs can be imagined for all types of genetic tests, from karyotyping to whole genome sequencing. They can be directly related to the genetic test carried out, such as a chromosomal abnormality related to a disorder not specifically under study, or they can be some other health or reproductive information discovered in the course of carrying out the test, such as misattributed paternity.
The present article is novel in using systematic review techniques in exploring the ethical issues involved with disclosing IFs, focusing on genetic testing. We do not distinguish between the different types of genetic tests, and only distinguish between the genetic context (research or clinical care) when this has a definite bearing on the argument used. Screening contexts are not included in the current review, as they introduce unique public health and policy concerns not so relevant in research or clinical care. A review has recently been published on genetic screening. ${ }^{9}$ The present review of the arguments that have been used in the ethical literature is of importance for all geneticists as they make decisions about the disclosure of IFs. It is of particular importance now, because new sequencing technologies such as whole genome and exome sequencing will produce IFs every time they are applied and the results analyzed. ${ }^{10}$ New sequencing technologies will only exacerbate the problem of IFs, because of the huge amounts of data they generate and the widely varying significance of the data, ranging from unknown to ambiguous to critical. ${ }^{11-13}$

\section{METHODS}

Aim

Systematic reviews of reasons are a relatively new form of systematic review, suitable for use in the field of reason-based bioethics. ${ }^{14,15}$ They aim to answer not an ethical question per se, as in some systematic reviews of argument-based clinical ethics literature, but rather the empirical question of which reasons have been given in the literature when a specific ethical question was addressed. The specific ethical question that the present article is interested in, is: should IFs arising in research and clinical genetics contexts be disclosed? The related empirical question is: which ethical reasons have been used to support and oppose the disclosure of IFs?

${ }^{1}$ Centre for Biomedical Ethics and Law, Catholic University of Leuven, Leuven, Belgium; ${ }^{2}$ Centre for Human Genetics, Catholic University of Leuven, Leuven, Belgium *Correspondence: GM Christenhusz, Centre for Biomedical Ethics and Law, Catholic University of Leuven, Kapucijnenvoer 35 , 3000 Leuven, Belgium, Tel: +32 16 3329 19; Fax: +32 163369 52; E-mail: gabrielle.christenhusz@med.kuleuven.be 
The aim of the present study was therefore to conduct a systematic review of the ethical reasons presented in the literature for and against the disclosure of IFs arising in research and clinical genetics contexts. Search terms and the inclusion and exclusion criteria that were applied to the resulting literature stemmed from this aim.

\section{Search methods}

The electronic databases Pubmed, Entrez, BioMedSearch and Hubmed were searched, using the search string ('incidental findings' and (Bioethics or Biomedical research/ethics or Ethics, Medical or Ethics, Research)). An advanced search of Web of Science was conducted using (TS $=$ (incidental findings) and TS $=$ (bioethics or biomedical research or ethics)), covering all years and citation databases. The results of each search were downloaded to Endnote, and duplicates discarded. These searches were conducted in December 2010. The Pubmed and Web of Science searches were saved and weekly electronic updates requested until September 2011.

In the first phase of searching, no mention was made of genetics in the search terms, as this excluded too many results. In the second phase of searching, various exclusion and inclusion criteria were applied, first by reading titles and abstracts, and then by reading the full text. Publications were included for systematic review if they presented ethical reasons for or against the disclosure of IFs arising in research and clinical genetics contexts. In line with the methodology for systematic reviews of reasons proposed by Sofaer and Strech, ${ }^{14}$ publications dealing with guidelines and regulations were excluded, as were those of a clinical or empirical nature, for instance on the frequency of IFs in a particular research or clinical field, and all empirical bioethical studies. Only publications dealing with ethical reasons were included. After finalizing which publications were to be included for systematic review, a few more publications were added as a result of the 'snowballing method'.

Only the term 'incidental findings' was used in the database searches, and not 'return of (individual) results' or any other related terms. Although we acknowledge that some of the literature on return of results also deals with the return of IFs, there are crucial differences between the two concepts, related to whether the finding falls inside or outside the domain or expertise of the researcher or clinician, and clear or ambiguous follow-up obligations. ${ }^{1}$ We therefore decided to restrict our current search to only those articles that dealt with IFs. We return to this choice below.

\section{Abstraction and synthesis of ethical arguments}

Beginning at the level of abstract (when present) and proceeding to the level of full text, we divided the ethical arguments into three categories: arguments in favor of disclosure; arguments opposed to disclosure; and arguments that caution about disclosure (Table 1). What is presented below does not include the number of times each argument is cited in the literature, in contrast to the methodology advocated by Strech and Sofaer. ${ }^{15}$ This is because we consider it irrelevant how many articles do or, more specifically, do not cite a particular argument. There is also no qualitative assessment made of the reviewed literature, as is the practice in systematic reviews of reasons. ${ }^{14}$ The discussion that follows presents our analysis and recommendations.

\section{RESULTS}

\section{Electronic database searches}

The electronic database searches resulted in a possible 229 references for systematic review, which were then read again more closely, and the inclusion and exclusion criteria detailed in the methods section applied. The full texts of the resulting 35 articles were read, and eventually 13 articles were accepted for systematic review (Figure 1). Of these 13, 9 came from the original electronic databases searches, ${ }^{3,8,12,16-21} 4$ from the 'snowballing method', $11,13,22,23$ and none from electronic updates between December 2010 and September 2011 from Pubmed and Web of Science.

\section{Reasons in favor of the disclosure of IFs}

The strongest reason in favor of the disclosure of an IF is its confirmed clinical utility and the possibility of treatment or prevention. $8,12,18,19,22,23$
This can justifiably be called the strongest reason, because all of the other reasons we identified in the literature, either for or against disclosure or offering caution, present themselves either in accord with or in contrast to confirmed clinical utility and the possibility of treatment or prevention. Interestingly, only the articles dealing with research mention this reason. The four articles dealing with clinical care do not mention it, giving only arguments against disclosure or offering caution. $3,11,16,20$ Some define clinical utility both in terms of what a medical professional would judge to be important clinically and what a research participant would judge to be important in terms of health or reproductive information. ${ }^{17}$ In certain circumstances, confirmed clinical utility is an argument strong enough to outweigh a stated preference for non-disclosure. For instance, if an IF from a minor is serious and treatable, thus having clinical utility, this can be an argument in favor of disclosure that overrides the right of parents or guardians to refuse disclosure. ${ }^{19}$ An example is the incidental discovery of a mutation in the $A P C$ gene related to familial adenomatous polyposis: this discovery can be acted on through early screening and detection of colonic and extracolonic tumors. ${ }^{19}$ Similarly, the clinical utility of IFs such as information about carrier status or present or future health risks for family members and other third parties can be an argument in favor of disclosure that overrides the right of the original subject of the genetic test to oppose disclosure. ${ }^{23}$

\section{Reasons opposing the disclosure of IFs}

A number of arguments are presented in the reviewed literature regarding circumstances in which IFs should not be disclosed. One cluster of arguments is simply the reverse of the confirmed clinical utility argument used to reason in favor of disclosure: if an IF has unlikely net benefit, it should not be disclosed. This can occur in the early stages of research, for instance, when it can be difficult to validate suspected IFs; several authors advise against disclosing IFs at such a stage, because of the risks of both over-treatment and undertreatment, unwarranted labeling and anxiety on the one hand or a false sense of security on the other hand. ${ }^{8,21}$ An example is the IF of a $\mathrm{CNV}$ associated with autism. Research on the connection between CNVs and autism is at too early a stage to be able to say anything certain about this IF at this stage, and if the IF is reported the result may be over-treatment and unwarranted anxiety. ${ }^{21}$ Such concerns are also valid in the clinic, if the significance of a particular IF is unclear. ${ }^{11}$ Because IFs are findings that fall outside the original aims of the study, it can be the case that they fall outside the expertise of the primary researcher or clinician. If it is impossible to find the necessary expertise to verify and assess an IF, this can be a valid argument against disclosure, though it is not the only relevant factor when deciding about disclosure. ${ }^{17}$

In addition, another cluster of arguments emphasize that what is at issue is net benefit: authors writing in a clinical setting argue that if the accompanying anxiety and possible discrimination and stigmatization from the point of view of the patient are perceived as greater than the potential clinical utility, this can argue against disclosure. ${ }^{3,11}$ An example is the IF that someone is at heightened risk of developing Alzheimer's disease. ${ }^{3}$ Furthermore, the potential but unlikely benefits accruing from disclosing an IF that is not so serious, untreatable, and/ or of uncertain significance may be so small that, in a research setting, it is better to respect the subject's right not to know and not disclose. ${ }^{12}$ Unlikely net benefit is a particular concern when minors are involved. Physical and psychological risks argue against the disclosure of any IFs without clear and proximate importance discovered in minors; these IFs could be late-onset conditions such 
Table 1 : Arguments in favor of the disclosure of IFs, arguments opposed to disclosure and arguments that caution about disclosure, presented in the reviewed literature. The overall conclusion of the article regarding disclosure is also stated.

\begin{abstract}
Author/s
and date

Reasons offered on the disclosure of IFS

Ali-Khan

et al, $2009^{11}$

Anti-disclosure: IFs discovered at an early stage of research; when the accompanying
anxiety and possible discrimination and stigmatization outweigh potential clinical utility; potential information overload; increased time and staff expertise needed meaning that not all IFs can be analyzed and communicated; physical and psychological risks associated with IFs without clear and proximate importance discovered in minors, for example lateonset conditions and non-medical conditions

Caution: respecting the subject's autonomy against unwanted paternalism, except in unanticipated situations; implications for family members, the community and relevant population groups
\end{abstract}

Botkin, $1995^{16}$

Caulfield

et al, $2008^{22}$

Cho, $2008^{17}$

Clayton, $2008^{18}$

Hens et al, $2011^{19}$

Kaye et al, $2010^{12}$

Kohane et al, $2006^{13}$

McGuire et al, $2008^{23}$

Parker and Majeske, $1995^{20}$

Tabor and Cho, $2007^{21}$

Van Ness, $2008^{8}$

Caution: respecting the subject's autonomy against unwanted paternalism, except in unanticipated situations when beneficence as judged by the medical professional should come first

Pro-disclosure: confirmed clinical utility and the possibility of treatment or prevention

Caution: implications for family members, the community and relevant population groups

Pro-disclosure: confirmed clinical utility and the existence of an effective intervention or prevention, for example information about susceptibility to environmental factors or reproductive information

Anti-disclosure: unavailability of the necessary expertise to verify and assess IFs; 'therapeutic misconception'; lack of an ongoing relationship between researcher and research participant when future IFs are involved

Caution: implications for family members, the community and relevant population groups

Pro-disclosure: confirmed clinical utility and the possibility of treatment or prevention Anti-disclosure: if research participants have not been prepared for the possibility of disclosure or the IF does not have grave consequences when future IFs are involved

Pro-disclosure: serious and treatable, which can override parental wishes for non-disclosure

Anti-disclosure: 'therapeutic misconception'; physical and psychological risks associated with IFs without clear and proximate importance discovered in minors, for example lateonset conditions and non-medical conditions

Pro-disclosure: confirmed clinical utility and the possibility of treatment or prevention

Anti-disclosure: the subject's right not to know coupled with an IF that is not so serious, untreatable and/or of uncertain significance

Caution: implications for family members, the community and relevant population groups

Anti-disclosure: information overload and too high follow-up times and costs, discouraging new forms of genetic testing

Pro-disclosure: confirmed clinical utility and the possibility of treatment or prevention, which can also argue for disclosure to family members and third parties, overriding the wishes of the original subject

Caution: implications for family members, the community and relevant population groups

Caution: respecting the subject's autonomy against compulsory disclosure; implications for family members, the community and relevant population groups

Anti-disclosure: IFs discovered at an early stage of research, because of the increased potential of harm; potential information overload

Pro-disclosure: confirmed clinical utility and the possibility of treatment or prevention

Anti-disclosure: IFs discovered at an early stage of research
Conclusion of the article

In the context of whole genome sequencing, disclosure of IFs should be done with caution

Disclosure of IFs should be done with caution

In favor of qualified disclosure, with more emphasis on favoring disclosure

In favor of qualified disclosure

Disclosure of future IFs should be done with caution

In the context of minors, disclosure of IFs should be done with caution

In favor of qualified disclosure

Disclosure of IFs should be done with caution, and the generation of IFs should be limited as much as possible

In favor of qualified disclosure, with special attention given

to a family-centered approach

Disclosure of IFs should be done with caution

Disclosure of IFs should be done with caution

In favor of qualified disclosure, especially in the early stages of genomic research 
Table 1 (Continued)

Author/s

and date

Reasons offered on the disclosure of IFs

Conclusion of the article

Wachbroit, $1998^{3}$ Anti-disclosure: when the accompanying anxiety and possible discrimination and stigma- In favor of qualified disclosure

tization outweigh potential clinical utility

Caution: implications for family members, the community and relevant population groups,

which may argue in favor of disclosure

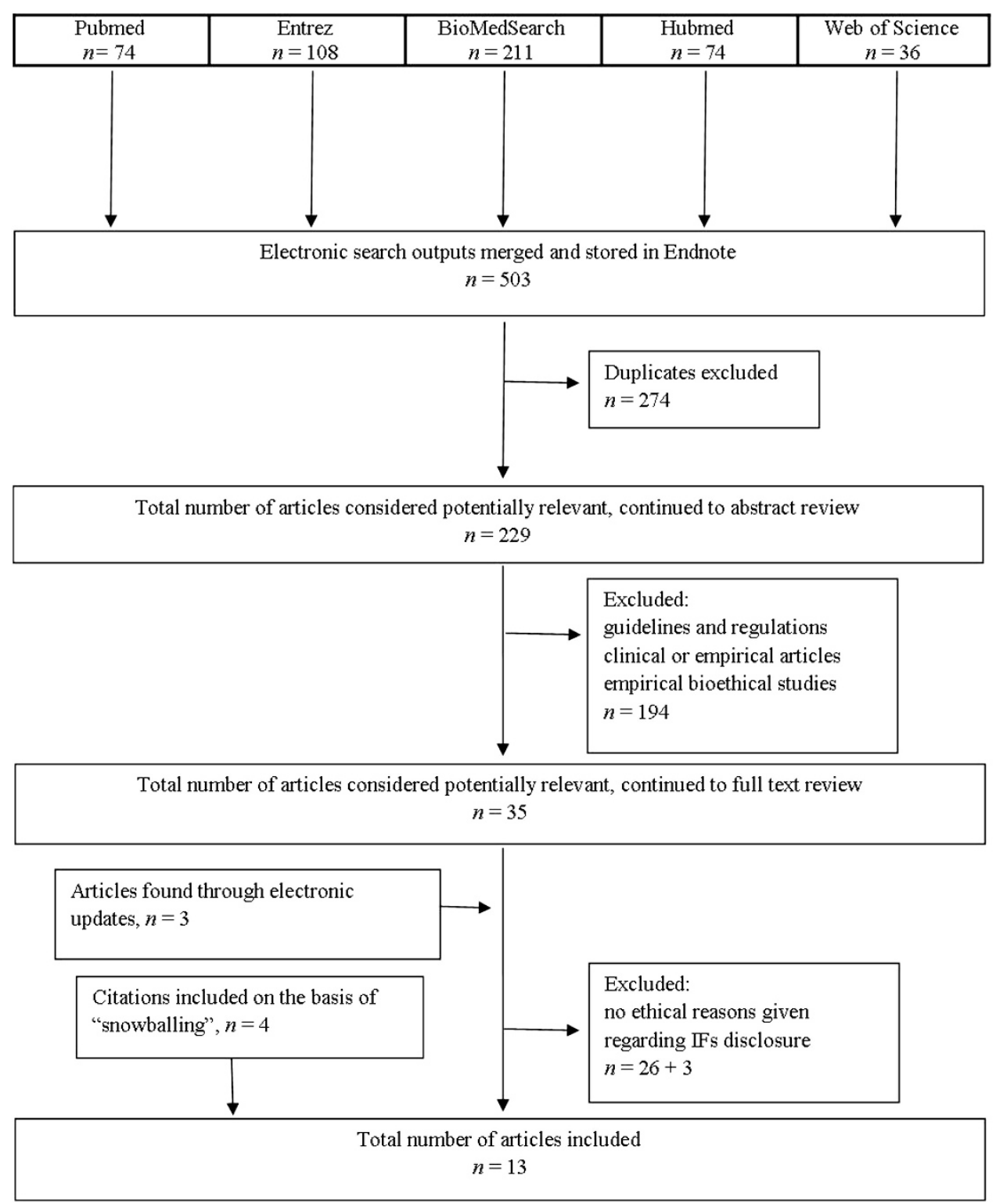

Figure 1 Results of the electronic database searches on the search string's 'incidental findings' and (Bioethics or Biomedical research/ethics or Ethics, Medical or Ethics, Research).

as breast cancer or non-medical conditions. ${ }^{11,19}$ Carrier status can be a case in point, if there is some confusion that being a carrier for a disease such as cystic fibrosis actually has an effect on the child's health. ${ }^{19}$
Another type of argument that appears in the reviewed literature on genetic and genomic research to reason against disclosure is the so-called 'therapeutic misconception': that is, if research participants learn that there is a policy of routinely following up and disclosing 
IFs, they might expect that their participation in the research will yield personal, clinical benefit to them, and that if no IFs are disclosed to them they are completely healthy. ${ }^{17,19}$

When exactly an IF is discovered can be an argument against disclosure. One author argues that the follow-up duties expected from researchers with respect to IFs discovered in the future on data collected now be somewhat limited, especially if the researcher does not have an ongoing relationship with the research participant. ${ }^{17}$ Another author contends that research participants must be prepared beforehand for the eventuality of disclosure, or the IF must have grave consequences, otherwise it does not seem fair to surprise the participant with unexpected disclosure. ${ }^{18}$

The potential of simply too much information, leading to followup times and costs that are too high, all of which can conflict with the scientific obligations of researchers, are another cluster of reasons arguing against disclosure. This cluster of reasons is also used by the reviewed literature to argue in favor of limiting the possibility of IFs, thereby making non-disclosure in effect inevitable. Information overload from research, when an overwhelming number of IFs have to be prepared for and communicated to the participant, can argue against disclosure. ${ }^{21}$ There are some fears that the sheer amount of IFs and the follow-up time and costs may discourage new forms of genetic research, an argument to limit the possibility of IFs. ${ }^{13}$ Whole genome sequencing is expected to exacerbate the problem of information overload, resulting in increasingly intricate and complex IFs. This is expected to cause difficulties in clinical genetics too, requiring increases in time and staff expertise with the consequence that not all IFs can be analyzed and communicated. ${ }^{11}$

With these potential difficulties in mind, some authors argue that studies should be designed to limit the potential number of IFs and false positives, which can be done by further research into overall disease prevalence and risk estimations, limiting the scope of genetic testing, and the educating of doctors in how to interpret IFs. ${ }^{13}$ One suggestion specifically for use in clinical genetics is the development of filtering systems that automatically make decisions about disclosure, to save both professionals and subjects being overwhelmed with the sheer amount of information produced by genetic testing in a diagnostic setting. ${ }^{11}$ These measures argue indirectly for non-disclosure of IFs by limiting the number of IFs that arise.

\section{Reasons cautioning the disclosure of IFs}

A third group of reasons presented in the reviewed literature does not argue directly for or against disclosure, but offers caution when making decisions about disclosure. One reason for caution when making decisions about disclosure in clinical settings is respect for the right not to know and the autonomy of the patient. There is general consensus that IFs with clinical utility should be disclosed, but there is also a concern that compulsory disclosure might harm respect for the autonomy of the patient. ${ }^{20}$ At the same time, respect for autonomy is complicated when unanticipated situations arise and it is too late to hypothetically ask the patient what they do and do not want to know. Some authors see respecting autonomy as an antidote to unwanted paternalism, while adding that when unanticipated situations and information arise, decisions about disclosure should be left to the professionals, because they are best able to judge what will be in the subject's best interests. ${ }^{11,16}$

A second reason for caution is that IFs, especially those arising from genetic testing, can have implications for close family members and for the community and relevant population groups. $3,11,12,17,20,22,23$ These implications could be to the advantage or disadvantage of third parties. For example, it would be to the advantage of other family members to learn that they may be susceptible to a severe drug reaction, ${ }^{17}$ while it would be to the disadvantage of all those with a particular genetic condition if an IF found during research and related to that specific condition led to discrimination and stigmatization. ${ }^{24}$ It is argued that family and community-related issues be discussed at the informed consent and ethics review stages. Subjects should be encouraged to include other family members in decisions about involvement in genetic testing and the handling of IFs, and if conflicts arise between the wishes of different family members, it is recommended that these be investigated by a research ethics consultation team, if one exists, and reviewed by an ethics review board. ${ }^{23}$

\section{DISCUSSION}

\section{General recommendations in the literature regarding the disclosure} of IFs

Two main types of recommendations are made in the literature regarding the disclosure of IFs, based on various ethical reasons (Table 1). One recommendation is in favor of what could be called qualified disclosure; that is, IFs should be disclosed except in special circumstances. One article, from a research context, was very much in favor of disclosure; ${ }^{22}$ others in favor of qualified disclosure mentioned reasons such as the unavailability of the necessary expertise to verify and assess IFs, the therapeutic misconception, unlikely net benefit and family concerns. ${ }^{3,8,12,17,23}$ A second type of recommendation is more hesitant: the disclosure of IFs should be done with caution. This recommendation is made in specific contexts such as whole genome sequencing and other new genetic technologies, genetic testing involving minors and IFs discovered in the future on data collected now. $3,11,13,18,19,21,23$ It is also made when balancing the autonomy of patients to make their own decisions against the superior expertise and knowledge of medical professionals. ${ }^{16,20}$

The starting point for the two recommendations is that IFs with confirmed clinical utility and having the possibility of treatment or prevention should be disclosed, and then exceptions are made. However, the breadth of terms such as 'treatment' and 'prevention' can be quibbled over. Does treatment only extend to clinical treatment? Or can it be interpreted as 'actionable', extending to information that can be acted upon in the making of decisions, such as decisions about reproductive choices or life insurance packages? Misattributed paternity is often cited as an IF that is not clinically significant but that many people would probably wish to have disclosed to them. ${ }^{25}$ On the other hand, Ross challenges the labeling of misattributed paternity as an 'incidental finding', as this might mask the serious moral accusation contained in such a finding. ${ }^{26}$ This is an example that raises several problems simultaneously: even if disclosure plans are drawn up for clinically significant, actionable IFs, different people will respond differently to the same finding, and it can be difficult to anticipate someone's response if the professional-subject relationship is relatively distant and impersonal, though this distance may be in line with the nature of the genetic test. Expressed another way, issues arise around how fixed definitions and protocols interact with respect for autonomy and professional roles.

One unanswered question is how to communicate these decisions and recommendations for the disclosure of IFs as efficiently as possible to patients and research participants, for their consent or dissent. Concrete proposals for how to achieve informed consent in practice are absent from the literature. If all possible examples of IFs and disclosure and follow-up options need to be discussed with the subject, this could take hours. ${ }^{27}$ This is one reason that some authors advocate the use of filtering systems or various governing structures 
to relieve professionals and subjects from the burden of information overload. ${ }^{11,12,22}$ Some degree of filtering is applied in any case in genetic sequencing, so that only the most likely disease-causing variants are analyzed. ${ }^{28,29}$ These filtering systems could also be set up to automatically filter out results known to be insignificant or not actionable, depending on the context. If they only filter in results known to be clinically significant and actionable, this would make the appearance of IFs almost, but not completely, impossible. Pleiotropic conditions or misattributed paternity, for example, could still conceivably be discovered. The question remains who decides what is clinically significant and actionable.

With such a filtering system, informed consent would then amount to consenting that others make a lot of the necessary decisions, and many of these decisions could be that many IFs simply do not come to the conscious attention of the clinicians/researchers or patients/ participants. This proposed application of informed consent conflicts with individual autonomy and privacy, ${ }^{12}$ but may be the best way to uphold the value of beneficence. ${ }^{20}$ If most people do not even know where genes can be found in the body or how genes are inherited, ${ }^{30}$ can those presenting at a genetics clinic or for genetics research really be expected to decide what is in their best interests in terms of the handling of IFs? A related issue surrounds those who have forgotten that a genetic test was performed on their DNA in the past, and this marks a difference between IFs arising in genetics and IFs arising in areas such as neurology and oncology. In the latter scenarios, people will at least remember undergoing a physical scan or biopsy. As we have seen above, some argue that it is better not to agitate people with questions about findings from genetic tests that they may have long forgotten about. ${ }^{18}$ Finally, it is interesting to note that genetic counseling arose as a reaction to the eugenics of the last century, so that people could make decisions about the use of genetic information without coercion or threats to their autonomy, and not because it was thought that genetic information was particularly dangerous or risky to an individual, their family or society. ${ }^{3}$ More research into the views of clinicians, researchers, patients and participants on how automatized filtering systems interact with their professional and individual autonomy appears to be a good way forward. We are in favor of automatized filtering systems that would save the time, money and expertise of medical professionals and subjects for IFs that really are clinically significant and actionable.

The research question for the present systematic review focuses on the ethical reasons presented in discussions on the disclosure of IFs. Few of the articles attempt a ranking of the ethical reasons. Exceptions are those that set respect for the subject's autonomy over the beneficence that compulsory disclosure tries to safeguard, ${ }^{20}$ and those that set beneficence as defined by the medical professionals over respect for the subject's autonomy. ${ }^{16}$ More work could be done in ranking the ethical reasons relevant when deciding about disclosure. An early article on categorizing genetic tests attempts just this. ${ }^{31}$ The article divides genetic tests into four types, based on the combination of clinical validity (present or absent) and the effectiveness and availability of treatment (present or absent). It is argued that the degree of clinical validity and the availability of an effective treatment emphasize in different ways concerns about respect for autonomy, risks, benefits, and how widely available testing and treatment are. Once the varying concerns are ranked, a plan for disclosure and for follow-up can be justified.

\section{Clinical care and research}

The majority of the articles included in this systematic review deal solely with researchers, and do not mention clinicians. One reason for this is that it is unclear whether 'incidental finding' is a useful term in clinical contexts, or if all results are actually included in the aim of clinical care. ${ }^{1,32}$ The goal of clinical care is the advancement of the patient's health-related welfare, and so it may be fairly easy to make decisions about disclosure because it is the patient's health that must take priority. ${ }^{16,25}$ This may be why none of the articles covering clinical care advise that IFs of confirmed clinical utility with treatment or prevention possibilities be disclosed to patients, while most of the articles covering research stress this point. In addition, the clinician is likely to have a good enough relationship with their patient that they will have the opportunity to discuss the possibility of IFs before a test or procedure is undertaken, or they will be able to anticipate what the patient's wishes regarding IFs will be even before asking. ${ }^{25}$

We have made a distinction, where relevant, between arguments made in research contexts and those made in clinical contexts. At the same time, IFs challenge the traditional research/clinical care divide by introducing clinical problems into research contexts. ${ }^{33}$ Clinicians owe their patients a duty of care, and this entails an obligation of using professional care in analyzing all information about a patient; not actively searching for IFs per se, but certainly recognizing what ' a professional of his or her training would ordinarily recognize.' Clinical researchers do not automatically fall under the category of clinician when they conduct clinical research, and expecting them to act completely like clinicians can cause problems because they are not in a clinician-patient relationship. ${ }^{33,34}$ The challenge in our opinion is to expand the role of researcher so that it includes a consideration of (clinical) IFs without jeopardizing the scientific enterprise.

\section{Is 'incidental findings' the best name?}

Part of the challenge in ethical reflection on IFs is the need for standardized terminology, and the question whether incidental findings' is indeed the best term. Debate about disclosing IFs comes out of the earlier debate about returning research results. The earlier debate arrived at various conclusions about checking analytic validity, clinical validity and clinical utility, the right not to know and the disclosure of results that are clinically significant and actionable. ${ }^{1,35,36}$ This has informed the IFs debate, for example that IFs that are clinically significant and actionable will be disclosed. However, there are two key differences between research results and IFs, which argue that the two be considered separately. ${ }^{1}$

One difference is that while research results will lie within the expertise of the researcher, IFs may not, so that interpreting IFs and deciding how to handle them may require outside expert help. The unavailability of outside expert help can be a reason not to disclose IFs. ${ }^{1,17,33}$ This difference in expertise also means that different professionals may identify different IFs based on the same data, depending on their particular specialty. A second difference is that the extent to which researchers should go to confirm the existence and severity of an IF is unclear, while this lack of clarity is simply not an issue with research results. On the basis of these differences, Wolf et $a l^{1}$ argue that IFs should be considered apart from the related issue of return of results, as we have done in this systematic review. However, not all authors agree, and it is possible that they may simply not have thought about it, spontaneously considering IFs as part of a larger reflection on return of results. This is a shortcoming in the search strategy employed in this systematic review. 'Return of results' or variations thereof were never included in the search strings, and so if an article included ethical reflection on IFs but did not include 'incidental findings' as a keyword, this article will not have been located except through the snowballing method. However, based on the reasoning above, we stand by our decision to 
maintain a clear distinction between research results and IFs in the current systematic review.

It is essential to make a clear distinction between research results and IFs. At the same time, ethical reflection on one can advantageously inform ethical reflection on the other. This is especially pertinent for those forms of genetic research, new forms of genetic sequencing, and all clinical contexts which blur the boundaries between research results and IFs, making what is 'incidental' unclear. It may be helpful to bring IFs back under the umbrella of '(research) results', and to deal with the two differences listed above (lying inside or outside one's expertise, and clear or ambiguous follow-up obligations) by expanding and clarifying the obligations expected of researchers or clinicians. One helpful development would be to harmonize guidelines and recommendations on research results and IFs regarding disclosure and follow-up procedures.

Another, complementary, option is to consider alternative names for 'incidental findings'. IFs is the term used in the current article because it is the keyword recognized by the search engines employed, but there was some discomfort expressed with the term in the literature. The most common complaint was that the adjective 'incidental' can minimize the significance of the finding, and is less than appropriate in describing certain potentially life-changing findings. ${ }^{25,37}$ The term 'unanticipated findings' is suggested and then rejected by Parker ${ }^{25}$ because, as she points out, the frequency of some 'unanticipated findings' can be estimated based on the known frequency in the population, and some research and clinical activities are so liable to generating these types of findings that they cannot really be called 'unanticipated'. The Health Council of the Netherlands uses the terms 'sought for findings' and 'unsought for findings' in a recent document on whole genome sequencing. ${ }^{38}$ These terms are attractive because they make use of the commonly accepted definition of 'incidental findings', related to the aim of a given study. However, one of the foundational tenets of science is that you only find what you are looking for. Something like HIV may be an 'incidental finding' under the present terminology, but it will only be discovered if an HIV test is carried out. In this sense, HIV is a 'sought for finding, even if found coincidentally in the broader context of genetic testing. An exception is pleiotropic conditions, when information about one gene related to one genetic condition also reveals information about other genetic conditions, and the other conditions could be considered 'unsought for' Nevertheless, even in this case someone would have to know that, for instance, the ApoE gene is implicated in cardiac disease and Alzheimer's disease; someone who does not know this will not make the IF. An IF must be 'found.' A genome sequence that is not analyzed cannot unveil IFs, just as an unread neurological or oncological scan cannot. Only an analyzed image or sequence can produce IFs. A final possibility for a name is 'off-target' results, ${ }^{27}$ though it remains to be seen how widespread this term might become. This points to the main problem with trying different terms for 'incidental findings': ultimately, the terminology must be standardized, so that it is clear when people are talking about the same thing in either scientific articles, guidelines, informed consent forms, or clinical or research reports. A term that accurately describes the phenomenon of 'incidental findings' but that is rarely used has its limitations.

One potential limitation of the articles included in this systematic review is that less than a third of them include a medical doctor as opposed to academics as a contributing author (4 out of 13 , on the basis of the institutions and qualifications listed for the authors). An academic with no practical experience of dealing with IFs with research participants or patients will be limited in how much they can say about the practical applicability of their ethics. Some of the authors may of course have previous practical experience, that is not apparent from the name of their current institution, and not all medical (MD) qualifications may have been listed. An additional further limitation of the articles is that all but two were written in North America. The apparent silence from the rest of the world may mean that they use a keyword other than 'incidental findings', or that they are not yet ethically interested in IFs. It would be interesting to investigate whether IFs are a specifically North American phenomenon, although the existence of this current European systematic review suggests otherwise.

\section{CONFLICT OF INTEREST}

The authors declare no conflict of interest.

\section{ACKNOWLEDGEMENTS}

This work was supported by FWO Flanders, project number G029107 and G.0594.09. K Devriendt is senior clinical investigator of the FWO-Vlaanderen and of the K.O.O.R U.Z.Leuven. We thank the anonymous reviewers for their valuable comments and suggestions.

1 Wolf S, Lawrenz F, Nelson C et al: Managing incidental findings in human subjects research: analysis and recommendations. J Law Med Ethics 2008; 36: 219-248.

2 Roof K, Gregorio DI, Kulko J, Palermino D: Incidental findings in a federally-sponsored cancer screening program. J Community Health 1999; 24: 305-312.

3 Wachbroit R: The question not asked: the challenge of pleiotropic genetic tests Kennedy Inst Ethics J 1998; 8: 131-144.

4 Lumbreras B, Donat L, Hernandez-Aguado I: Incidental findings in imaging diagnostic tests: a systematic review. Br J Radiol 2010; 83: 276-289.

5 Morris Z, Whiteley WN, Longstreth WT et al: Incidental findings on brain magnetic resonance imaging: systematic review and meta-analysis. BMJ 2009; 339: b3016.

6 Siddiki H, Fletcher JG, McFarland B et al: Incidental findings in CT colonography: literature review and survey of current research practice. J Law Med Ethics 2008; 36: 320-331, 213

7 Miller F, Hayeems R, Bombard $Y$ et al: Clinical obligations and public health programmes: healthcare provider reasoning about managing the incidental results of newborn screening. J Med Ethics 2009; 35: 626-634.

8 Van Ness B: Genomic research and incidental findings. J Law Med Ethics 2008; 36 292-297.

9 Burke W, Tarini B, Press NA, Evans JP: Genetic screening. Epidemiol Rev 2011; 33 148-164.

10 Evans JP: Looking ahead, looking behind. Introduction. Genet Med 2011; 13: 177-178.

11 Ali-Khan S, Daar A, Shuman C, Ray P, Scherer S: Whole genome scanning: resolving clinical diagnosis and management amidst complex data. Pediatr Res 2009; 66 : 357-363.

12 Kaye J, Boddington P, de Vries J, Hawkins N, Melham K: Ethical implications of the use of whole genome methods in medical research. Eur J Hum Genet 2010; 18: 398-403.

13 Kohane I, Masys D, Altman R: The incidentalome: a threat to genomic medicine. JAMA 2006; 296: 212-215.

14 Sofaer N, Strech D: The need for systematic reviews of reasons. Bioethics 2011 Epub ahead of print.

15 Strech D, Sofaer N: How to write a systematic review of reasons. J Med Ethics 2012 38: 121-126.

16 Botkin J: Genetic counseling: making room for beneficence. J Clin Ethics 1995; 6: 182-184.

17 Cho M: Understanding incidental findings in the context of genetics and genomics. J Law Med Ethics 2008; 36: 280-285.

18 Clayton E: Incidental findings in genetics research using archived DNA. J Law Med Ethics 2008; 36: 286-291.

19 Hens K, Nys H, Cassiman J, Dierickx K: The return of individual research findings in paediatric genetic research. J Med Ethics 2011; 37: 179-183.

20 Parker L, Majeske R: Incidental findings: patients' knowledge, rights, and preferences. J Clin Ethics 1995; 6: 176-179.

21 Tabor H, Cho M: Ethical implications of array comparative genomic hybridization in complex phenotypes: points to consider in research. Genet Med 2007; 9: 626-631.

22 Caulfield T, McGuire A, Cho M et al: Research ethics recommendations for whole genome research: consensus statement. PLoS Biology 2008; 6: e73.

23 McGuire A, Caulfield T, Cho M: Research ethics and the challenge of whole-genome sequencing. Nat Rev Genet 2008; 9: 152-156.

24 Rotimi CN, Marshall PA: Tailoring the process of informed consent in genetic and genomic research. Genome Med 2010; 2: 20.

25 Parker L: The future of incidental findings: should they be viewed as benefits? J Law Med Ethics 2008; 36: 341-351 
26 Ross LF: Good ethics requires good science: why transplant programs should not disclose misattributed parentage. Am J Transplant 2010; 10: 742-746.

27 Mayer AN, Dimmock DP, Arca MJ et al: A timely arrival for genomic medicine. Genet Med 2011; 13: 195-196.

28 Bamshad MJ, Ng SB, Bigham AW et al: Exome sequencing as a tool for Mendelian disease gene discovery. Nat Rev Genet 2011; 12: 745-755.

29 Cooper GM, Shendure J: Needles in stacks of needles: finding disease-causal variants in a wealth of genomic data. Nat Rev Genet $2011 ; 12$ : 628-640.

30 Lanie AD, Jayaratne TE, Sheldon JP et al: Exploring the public understanding of basic genetic concepts. J Genet Couns 2004; 13: 305-320.

31 Burke W, Pinsky LE, Press NA: Categorizing genetic tests to identify their ethical, legal, and social implications. Am J Med Genet 2001; 106: 233-240.

32 Miller F, Mello M, Joffe S: Incidental findings in human subjects research: what do investigators owe research participants? J Law Med Ethics 2008; 36: 271-279.
33 Wolf S, Paradise J, Caga-anan C: The law of incidental findings in human subjects research: establishing researchers' duties. J Law Med Ethics 2008; 36: 361-383.

34 Wolf S: Introduction: the challenge of incidental findings. J Law Med Ethics 2008; 36: $216-218$

35 Knoppers B, Joly Y, Simard J, Durocher F: The emergence of an ethical duty to disclose genetic research results: international perspectives. Eur J Hum Genet 2006; 14: 1170-1178.

36 Ravitsky V, Wilfond B: Disclosing individual genetic results to research participants. AJOB 2006; 6: 8-17.

37 Sharp H, Orr R: When 'minimal risk' research yields clinically-significant data, maybe the risks aren't so minimal. AJOB 2004; 4: W32-W36.

38 Health Council of the Netherlands: The 'thousand-dollar genome': an ethical exploration. Monitoring Report Ethics and Health. The Hague: Centre for Ethics and Health, 2010. 